\title{
Highly sensitive troponin and diagnostic accuracy in acute myocardial infarction
}

\author{
Elisa Ceriani • Anna Maria Rusconi • \\ Gruppo di Autoformazione Metodologica (GrAM)
}

Received: 14 May 2012/ Accepted: 21 June 2012/Published online: 6 July 2012

(C) SIMI 2012

\section{Background}

Chest pain is one of the most common reasons for which patients seek care in the emergency department. When evaluating these patients, much effort is made to recognize cardiac causes of chest pain, in particular acute coronary syndrome (ACS), and to identify high-risk patients who may benefit from more aggressive treatments. Cardiac troponins play a pivotal role for this purpose. The diagnosis of acute myocardial infarction (AMI) is based mainly on an elevated cardiac troponin level exceeding the 99th percentile; this since 2000, when the joint committee of the European Society of Cardiology and the American College of Cardiology (ESC/ACC) published a new definition of AMI that for the first time officially included these biomarkers [1].

Recently introduced high-sensitivity troponin assays have improved the early diagnosis of acute myocardial infarction, and have a pivotal role in diagnosis, risk stratification, and management of patients with acute coronary syndromes [2-4], but their ideal cut-off and critical changes are yet to be established. Moreover, in clinical practice, the use of troponin high sensitivity is likely to increase the number of false-positive results.

E. Ceriani $(\bowtie)$

Medicina Interna I, Dipartimento di Emergenza Urgenza, Pronto Soccorso e Medicina d'Urgenza, Ospedale Luigi Sacco, Università degli Studi di Milano, Milan, Italy

e-mail: celisa78@virgilio.it

\section{A. M. Rusconi}

Pronto Soccorso e Medicina d'Urgenza,

Dipartimento di Emergenza Urgenza,

Ospedale Civile SM della Scaletta,

Imola (BO), Italy

\section{Summary}

The study of Keller and co-workers [5] compares the diagnostic performance of a novel highly sensitive troponin I (hsTnI) assay (Architect STAT High Sensitivity Troponin; Abbott Diagnostics) and a contemporary troponin I (cTnI) assay (Architect STAT) for diagnosing AMI at admission and at 3 h. 1818 consecutive patients with suspected acute coronary syndrome were enrolled from the chest pain units of three hospitals in Germany during 2007 and 2008. Four hundred and thirteen patients $(23 \%)$ received a diagnosis of AMI; 56 patients with AMI (14\%) presented with ST-segment elevation. Using a diagnostic cut-off troponin concentration representing the 99th percentile of a reference population, hsTnI at admission has a sensitivity of $82 \%$ and a negative predictive value (NPV) of $95 \%$ for AMI, and cTnI has a sensitivity of $79 \%$ and an NPV of $94 \%$. Sensitivity and NPV at $3 \mathrm{~h}$ for both assays is 98 and $99 \%$, respectively. Lowering the hsTnI cut-off at "level of detection", at admission the new assay shows a sensitivity and NPV of $100 \%$, with specificity of $35 \%$. Combining the $99^{\text {th }}$ percentile cut-off at admission with the relative change at $3 \mathrm{~h}$ and considering relative changes significant if more than $266 \%$, yields specificity and positive predictive values (PPV) of 100 and $96 \%$ for both assays. Considering relative changes significant if more than $50 \%$, the hsTnI specificity and positive predictive values are, respectively, 99 and $94 \%$ for hsTnI.

The authors conclude that "among patients with suspected acute coronary syndrome, hsTnI or cTnI determination 3 hours after admission may facilitate early rule-out of AMI. A serial change in hsTnI or cTnI levels from admission (using the 99th percentile diagnostic cut-off value) to 3 hours after admission may facilitate an early diagnosis of AMI". 


\section{Strengths of the study}

- This study tried to face a clinically relevant problem (AMI diagnosis with biomarkers).

- Many explorative analyses were made with different troponin serial changes cut-off values to investigate variations in diagnostic accuracy.

\section{Weakness of the study}

- "Patients with acute angina pectoris or equivalent symptoms", was an inclusion criterion, but its interpretation may be subjective. However, almost all the studies regarding ACS report a similar definition of included patients.

- A large part of this study is devoted to explorative analysis aimed at finding out the ideal threshold of troponin change, in order to optimize specificity. This feature is interesting, but conclusions cannot be directly used for clinical practice precisely because they were based on an exploratory analysis. Further studies are needed to possibly include them in clinical practice.

- Both the index test and reference standard included a change in troponin level over time (although the index test was hsTnI and cTnI while reference standard included inhouse troponin, with investigators blinded to index text results). This fact, as declared by the authors, may overestimate the diagnostic accuracy of the index test.

\section{Question marks}

- While it is clear from this study that serial troponin measurements are pivotal for specificity improvement, the ideal critical changes values to be used in clinical practice do not emerge from the manuscript. The authors explore many possible cut-off values of troponin increase. The $266 \%$ is the optimal cut-off for rule as derived from the ROC curve, because it has a specificity of $99 \%$, but at the expense of low sensitivity (not quantified in the text, however, the authors report a sensitivity of $35 \%$ using a cut-off of $250 \%$ ). Lowering the cut-off to $50 \%$ keep a specificity very high $(99.1 \%)$ with a sensitivity of $50 \%$. Moreover, for a better clinical interpretation of study results, it would have been interesting to also report the values of the Likelihood Ratio, which allow combination of pretest probability with test results, since PPV and NPV depend on the prevalence.

- The vast majority of patients came to ED with more than $3 \mathrm{~h}$ of chest pain. In $25 \%$ of the patients, an AMI could have been ruled out at admission by means of an undetectable troponin: it would be interesting to know how long the symptoms had been present in this group.

- It would be interesting to know how many patients had severe renal impairment, and how this condition may have influenced results.

- Not all patients received the index test, and apparently the entire troponin curve is not available for every patient: it would be interesting to know if some patients were discharged prematurely.

- The setting of this study was chest pain units. In fact, AMI prevalence is high $(23 \%)$ probably reflecting a high coronary risk population. It would be interesting to know if the high-sensitivity troponin assays have the same accuracy in other settings (external validity).

- Consider also the "level of detection" cut-off (i.e., undetectable troponin) for hsTnI at admission that could exclude AMI diagnosis in $25 \%$ of the patients with a sensitivity of $100 \%$. If validated by further studies, this strategy might be helpful to identify patients who are eligible for a safe early discharge from the emergency department without other tests.

\section{Clinical bottom line}

- HsTnI or cTnI assay shows a similar diagnostic performance. For patients with suspected ACS, negative results (considering the $99 \mathrm{t}^{\mathrm{h}}$ percentile cut-off) at $3 \mathrm{~h}$ after emergency department admission can be used to rule out AMI with a NPV of $99 \%$ for both assays.

- An undetectable hsTnI at admission could select patients who will not benefit from prolonged observation and troponin serial testing. However, for this point, validation studies are certainly needed to prove safety and feasibility of this strategy.

- Relative changes in assay results from admission to $3 \mathrm{~h}$ can be used to facilitate an early "rule in" strategy.

Acknowledgments The study was partially funded by the manufacturer of the assays, which however had no role in the design and conduct of study, management of data, and drafting the manuscript.

Conflict of interest None.

\section{References}

1. The Joint European Society of Cardiology/American College of Cardiology Committee. Myocardial infarction redefined-a consensus document of the Joint European Society of Cardiology/ American College of Cardiology Committee for the redefinition of myocardial infarction. Eur Heart J. 2000;21:1502-1513 
2. Keller T, Zeller T, Peetz D, Tzikas S, Roth A, Czyz E, Bickel C, Baldus S, Warnholtz A, Fröhlich M, Sinning CR, Eleftheriadis MS, Wild PS, Schnabel RB, Lubos E, Jachmann N, Genth-Zotz S, Post F, Nicaud V, Tiret L, Lackner KJ, Munzel TF, Blankenberg S (2009) Sensitive troponin I assay in early diagnosis of acute myocardial infarction. N Engl J Med 361:868-877

3. Giannitsis E, Becker M, Kurz K, Hess G, Zdunek D, Katus HA (2010) High-sensitivity cardiac troponin T for early prediction of evolving non-ST-segment elevation myocardial infarction in patients with suspected acute coronary syndrome and negative troponin results on admission. Clin Chem 56:642-650
4. Lindahl B, Venge P, James S (2010) The new high-sensitivity cardiac troponin $\mathrm{T}$ assay improves risk assessment in acute coronary syndromes. Am Heart J 160:224-229

5. Keller T, Zeller T, Ojeda F, Tzikas S, Lillpopp L, Sinning C, Wild P, Genth-Zotz S, Warnholtz A, Giannitsis E, Möckel M, Bickel C, Peetz D, Lackner K, Baldus S, Münzel T, Blankenberg S (2011) Serial changes in highly sensitive troponin I assay and early diagnosis of myocardial infarction. JAMA 306(24):2684-2693 